\title{
Skin perfusion pressure predicts mortality in hemodialysis patients: long term follow-up
}

\author{
Nanami Kida ${ }^{1 *}$, Shunro Ageta ${ }^{2}$, Yasunori Tsujimoto², Kiyoko Maehara², Masayuki Nagahara², \\ Yoshinosuke Hamada ${ }^{1}$ and Nariaki Matsuura ${ }^{1}$
}

\begin{abstract}
Background: Peripheral artery disease (PAD) is not only a serious complication but also a risk factor to predict prognosis in the patients with hemodialysis (HD). Ankle-brachial blood pressure index (ABI) has been widely used for detection of PAD. Skin perfusion pressure (SPP) was used for early detection of PAD by measurement of microcirculation of subcutaneous tissue. We studied the effectiveness of ABI and SPP to predict prognosis of HD patients.
\end{abstract}

Methods: Both SPP and ABI were measured in 45 HD outpatients in Nagahara Hospital. The patients were followed with a mean observational period of 44.4 months to evaluate the association of ABI or SPP value with any-cause mortality.

Results: The median ABI and SPP values in all patients were 1.15 and $70.0 \mathrm{mmHg}$, respectively, and the patients were classified into two groups with the median value used as the cutoff points. In Kaplan-Meier analysis, the lower SPP group showed significantly worse survival curve than the higher SPP group while there were no differences between ABI groups. Cox proportional hazards model demonstrated SPP values are independent and significant risk factors for mortality.

Conclusions: These results suggest evaluation of PAD by SPP measurement is useful to predict prognosis of HD patients.

\section{Background}

The annual mortality in chronic dialysis patients in Japan is less than $10 \%$ although the age of the patients has become higher and the proportion of the patients with long-term dialysis has increased [1]. The frequent causes of death in the hemodialysis patients are heart failure and cardiovascular diseases [2]. The prevalence of heart disease and vascular disease is expected to rise with increased elderly patients, increased patients with diabetes mellitus, and increased long HD patients [3, 4]. Thus, indicators should be required to predict heart failure and cardiovascular diseases in HD patients.

Peripheral artery disease (PAD) is a common complication in the hemodialysis patients $[5,6]$. The patients

\footnotetext{
* Correspondence: nanami@cam.hi-ho.ne.jp

1 Department of Molecular Pathology, Osaka University Graduate School of Medicine and Health Science, 1-7, Yamadaoka, Suita, Osaka 565-0871, Japan Full list of author information is available at the end of the article
}

with PAD have been reported to be susceptible to coronary heart disease and congestive heart failure as well as foot lesion in many cohort studies [7]. High prevalence of PAD is associated with a number of factors including increasing number of HD patients from diabetes, HD induction at older age, and complication of atherosclerosis due to abnormal calcium metabolism at the preserved stage of renal failure [8]. Some reports suggest renal insufficiency itself is an independent risk factor of PAD in the HD patients with or without diabetes [9].

Therefore, PAD is not only a serious complication of HD but also a risk factor to predict cardiovascular disease. Thus, early diagnosis of PAD is an important issue for the hemodialysis patients. Most HD patients usually do not have any symptoms at the initial stage of PAD, and early detection for PAD is pretty difficult [10]. Some patients undergo amputation of the lower extremities as the diagnosis of PAD is delayed. Amputation does not 
only decrease the quality of life (QOL) of the patients but also influence prognosis since it frequently causes critical infectious disease leading to death $[11,12]$. The patients with PAD have been reported to have poor prognosis due to high mortality rate from cardiovascular disease and high complication rate with other vascular diseases such as cerebrovascular disease [13].

Ankle-brachial blood pressure index (ABI) is a noninvasive diagnostic tool for early detection of PAD by measuring blood pressures in both the upper and lower extremities [14]. ABI is widely utilized for assessment of an artery of the lower extremities since ABI is easily performed and correlated with angiographic findings of an artery of the lower limb [15]. On the other hand, some reports suggest ABI is not sufficient for the HD patients with severe blood vessel calcification or advanced arteriosclerosis to be diagnosed as PAD [10, 16].

Recently, skin perfusion pressure (SPP) was used to detect PAD by Laser Doppler method. Since the skin circulation regulated by sympathetic nerves is easily detected by SPP, the condition of microcirculation can be accurately evaluated by SPP [17]. SPP is also applicable to the patients with edema or severe blood vessel calcification cases as SPP detects circulation of the subcutaneous tissue. SPP has been used for assessment of wound healing in the field of dermatology [18]. There are some reports that SPP can evaluate ischemic condition of the lower extremities which cannot be assessed by ABI [19]. In this study, both ABI and SPP are measured in the HD patients and their association with prognosis is analyzed.

\section{Methods}

This study is a retrospective analysis for 45 consecutive outpatient hemodialysis cases in Nagahara Hospital in November to December 2006 who were submitted to evaluation of the ABI and SPP. Clinical and biochemical data were utilized, including age, gender, duration of hemodialysis, hemoglobin, calcium, phosphate, parathyroid hormone (PTH), and albumin. Cardiac function by echocardiography was also applied. The patients who cannot walk on their own or underwent lower limb amputation were excluded in this study.

Both SPP and ABI were measured in all the patients prior to the start of hemodialysis. ABI was determined by form/ABI (OMRON COLIN, Japan). The systolic blood pressures were measured at the brachial artery of the upper limb without the vascular access and at the posterior tibial arteries of both ankles in the patients resting in supine position for $5 \mathrm{~min}$. ABI was calculated from the ratio of the ankle systolic pressure to the brachial one. SPP was evaluated by PAD3000 (KANEKA, Japan) at the sole of both feet of the patients in supine position. Both measurements were repeated three times, and the average values were defined.

The patients were followed between November 2006 and December 2010 with a mean observational period of 44.4 months to evaluate the association of ABI or SPP value with any-cause mortality.

All data are described as the mean $\pm \mathrm{SD}$, and Student's $t$ test was used for statistical analysis. Kaplan-Meier analysis was performed to analyze survival curve. Univariate and multivariate analyses were performed by logistic regression analysis in order to identify risk factors for mortality. A receiver operating characteristic (ROC) analysis was performed to estimate the cutoff point, sensitivity, and specificity. For all the comparison and statistical tests, $p$ value less than 0.05 was considered as statistically significant. All analyses were performed with the use of Dr. SPSS software version 11.

\section{Results}

The clinical and biochemical characteristics of the patients are shown in Table 1. Average age was $62.7 \pm$

Table 1 Characteristics of the study participants

\begin{tabular}{|c|c|}
\hline \multicolumn{2}{|l|}{ All patients $(n=45)$} \\
\hline Age (years) & $62.7 \pm 12.4$ \\
\hline Gender (m/f) & $31: 14$ \\
\hline Duration of hemodialysis (years) & $7.3 \pm 5.1$ \\
\hline Quantity of blood flow (ml/min) & $202.7 \pm 24.4$ \\
\hline Dialysis time (h) & $4.0 \pm 0.4$ \\
\hline \multicolumn{2}{|l|}{ Primary cause of ESKD, $n(\%)$} \\
\hline Diabetic nephropathy & $17(38.0)$ \\
\hline Chronic glomerulonephritis & $17(38.0)$ \\
\hline Nephrosclerosis & $2(4.4)$ \\
\hline Polycystic kidney disease & $2(4.4)$ \\
\hline Unknown and others & $7(15.6)$ \\
\hline Current smoking, $n(\%)$ & $5(11.1)$ \\
\hline \multicolumn{2}{|l|}{ Medication, $n$ (\%) } \\
\hline Statin & $5(11.5)$ \\
\hline Cilostazol & $5(8.9)$ \\
\hline Hemoglobin (g/dl) & $10.1 \pm 1.0$ \\
\hline Albumin (f/dl) & $4.1 \pm 0.4$ \\
\hline $\mathrm{Ca}(\mathrm{mg} / \mathrm{dl})$ & $9.4 \pm 0.7$ \\
\hline$P(\mathrm{mg} / \mathrm{dl})$ & $5.1 \pm 1.2$ \\
\hline BUN (mg/dl) & $69.3 \pm 12.4$ \\
\hline Cre (mg/dl) & $10.9 \pm 2.0$ \\
\hline i-PTH (pg/dl) & $176.9 \pm 112.0$ \\
\hline $\operatorname{LVD}(\mathrm{mm})$ & $51.1 \pm 5.1$ \\
\hline LVEF (\%) & $66.6 \pm 9.0$ \\
\hline
\end{tabular}

LVD left ventricular end-diastolic diameter, LVEF left ventricular ejection fraction, $i$-PTH intact PTH 
12.4 years (32-93), and 31 of the patients $(68.9 \%)$ were male. The mean dialysis duration was $7.3 \pm 5.1$ years $(0.2-21.5)$, and diabetes was the primary disease for HD in 17 patients $(38.0 \%)$ and currently smoking 5 patients (11.1\%). Some circulatory drugs were medicated: antihypertensive for 37 patients, statin for 5 patients, and cilostazol for 4 patients. In biochemical analysis, serum calcium was $9.4 \pm 0.7 \mathrm{mg} / \mathrm{dl}$, serum phosphate was $5.1 \pm$ $1.2 \mathrm{mg} / \mathrm{dl}$, serum albumin was $4.1 \pm 0.4 \mathrm{~g} / \mathrm{dl}$, and hemoglobin was $10.1 \pm 1.0 \mathrm{~g} / \mathrm{dl}$. Echocardiographic values showed left ventricular end-diastolic diameter (LVDd) at $51.1 \pm 5.1 \mathrm{~mm}$, left ventricular ejection fraction (LVEF) at $66.6 \pm 9.0 \%$, and left atrial dimension (LAD) at $37.4 \pm 5.2 \mathrm{~mm}$.

Mean ABI and SPP values in all patients were $1.1 \pm 0.2$ (median 1.15) and $64.7 \pm 23.7 \mathrm{mmHg}$ (median 70.0), respectively. According to $\mathrm{ROC}$ analysis, the cutoff value of ABI was 1.11 (sensitivity, 71\%; specificity, 72\%), SPP was $61.8 \mathrm{mmHg}$ (sensitivity, 71\%; specificity, 65\%). Although in previous study $40 \mathrm{mmHg}$ was used for cutoff point of SPP, $61.8 \mathrm{mmHg}$ of the cutoff value showed better sensitivity and specificity according to ROC analysis in our study (sensitivity $83.5 \%$ and specificity $18.2 \%$ in $40 \mathrm{mmHg}$ and sensitivity $26.6 \%$ and specificity $81.8 \%$ in $80 \mathrm{mmHg}$ ). Since both ABI and SPP cutoff values are similar to median values, we analyzed $A B I$ and SPP for various factors by using median values. Patients were classified into two groups for ABI or SPP results, with the median value used as the cutoff, and two groups were compared for their characteristics.

Age was significantly different between the higher and lower SPP groups, although there was no difference in dialysis duration, presence of diabetes, serum albumin, hemoglobin, serum phosphate, serum calcium, LVDd, LVEF, or LAD in two groups (Table 2). On the other hand, age and intact PTH were significantly different between the higher and lower ABI groups, although there was no difference in dialysis duration, presence of diabetes, serum albumin, hemoglobin, serum phosphate, serum calcium, LVDd, LVEF, or LAD in two groups (Table 2).

Eleven patients $(24.4 \%)$ died during the observation period. All the causes of deaths were circulatory diseases: heart failure in eight cases $(72.7 \%)$, acute myocardial infarction in two patients (18.2\%), and cerebrovascular disease in one patient (9.1\%). Mortality rate was significantly higher in the lower SPP group than that in the higher one $(p<0.05)$ while there was no statistical difference between the higher and lower ABI groups.

Kaplan-Meier analysis was performed for survival curve to evaluate the ABI and SPP values to predict prognosis (Fig. 1). While no significant difference was observed in the survival between the lower and higher ABI groups, the lower SPP group revealed significantly worse prognosis than the higher SPP group $(p<0.01)$.

In the univariate analysis, the predictive variables for all-caused deaths included age, diabetic status, SPP, ABI, LVEF, and albumin $(p=0.00,0.03,0.02,0.04,0.00$, and 0.04 , respectively). A multivariate cox proportional hazards model demonstrated SPP, LVEF, and diabetic status as significantly strong risk factors to all-caused death $(p=0.02,0.00$ and 0.05 , respectively). On the other hand, age, $\mathrm{ABI}$, and albumin were not independent risk factors for mortality (Table 3 ).

Table 2 Comparison of SPP and ABI lower and higher group in HD patients

\begin{tabular}{|c|c|c|c|c|c|c|c|}
\hline & $\begin{array}{l}\text { SPP }<70 \\
(n=22)\end{array}$ & $\begin{array}{l}\text { SPP } \geq 70 \\
(n=23)\end{array}$ & $p$ value & & $\begin{array}{l}\mathrm{AB} \mid<1.15 \\
(n=23)\end{array}$ & $\begin{array}{l}\mathrm{ABI} \geq 1.15 \\
(n=22)\end{array}$ & $p$ value \\
\hline Gender (male to female) & 13:09 & 18:05 & & Gender (male to female) & 15:08 & 16:06 & \\
\hline Age (years) & $66.8 \pm 14.5$ & $58.7 \pm 8.8$ & 0.04 & Age (years) & $68.0 \pm 11.4$ & $57.7 \pm 11.5$ & 0.01 \\
\hline HD duration (years) & $8.1 \pm 6.1$ & $6.6 \pm 4.0$ & 0.32 & HD duration (years) & $8.3 \pm 6.2$ & $6.4 \pm 3.9$ & 0.56 \\
\hline $\mathrm{DM}, n(\%)$ & $10(59.0 \%)$ & $7(41.0 \%)$ & 0.31 & $\mathrm{DM}, n(\%)$ & $11(64.7)$ & $6(53.3)$ & 0.09 \\
\hline Current smoking, $n(\%)$ & $3(60.0 \%)$ & $2(40.0 \%)$ & 0.91 & Current smoking, $n(\%)$ & $4(60.0 \%)$ & $1(40.0 \%)$ & 0.91 \\
\hline Statin, $n(\%)$ & $3(60.0 \%)$ & $2(40.0 \%)$ & 0.65 & Statin, $n(\%)$ & $3(60.0 \%)$ & $2(40.0 \%)$ & 0.66 \\
\hline Cilostazol, n (\%) & $3(75.0 \%)$ & $1(25.0 \%)$ & 0.46 & Cilostazol, n (\%) & $3(75.0 \%)$ & $1(25.0 \%)$ & 0.44 \\
\hline $\mathrm{Hb}(\mathrm{g} / \mathrm{dl})$ & $10.2 \pm 1.0$ & $10.0 \pm 1.0$ & 0.62 & $\mathrm{Hb}(\mathrm{g} / \mathrm{dl})$ & $10.2 \pm 0.9$ & $10.0 \pm 1.0$ & 0.62 \\
\hline Alb (g/dl) & $4.1 \pm 0.4$ & $4.2 \pm 0.3$ & 0.39 & Alb (g/dl) & $4.1 \pm 0.3$ & $4.1 \pm 0.5$ & 0.39 \\
\hline $\mathrm{BUN}(\mathrm{mg} / \mathrm{dl})$ & $68.5 \pm 12.1$ & $70.1 \pm 12.5$ & 0.65 & BUN (mg/dl) & $66.9 \pm 12.4$ & $71.6 \pm 11.8$ & 0.19 \\
\hline $\mathrm{Cr}(\mathrm{mg} / \mathrm{dl})$ & $10.3 \pm 1.9$ & $12.0 \pm 2.1$ & 0.01 & $\mathrm{Cr}(\mathrm{mg} / \mathrm{dl})$ & $10.8 \pm 2.1$ & $11.4 \pm 2.2$ & 0.38 \\
\hline$P(\mathrm{mg} / \mathrm{dl})$ & $4.9 \pm 1.2$ & $5.2 \pm 1.2$ & 0.43 & $P(\mathrm{mg} / \mathrm{dl})$ & $5.1 \pm 1.1$ & $5.1 \pm 1.3$ & 0.94 \\
\hline i-PTH (pg/dl) & $170.4 \pm 93.5$ & $182.0 \pm 129.7$ & 0.71 & i-PTH (pg/dl) & $213.1 \pm 134.8$ & $142.3 \pm 74.4$ & 0.03 \\
\hline LVDd (mm) & $50.5 \pm 5.1$ & $51.8 \pm 5.1$ & 0.41 & LVDd (mm) & $50.9 \pm 4.7$ & $51.4 \pm 5.4$ & 0.71 \\
\hline LVEF (\%) & $64.6 \pm 7.1$ & $68.5 \pm 10.2$ & 0.15 & LVEF (\%) & $64.6 \pm 9.3$ & $68.6 \pm 8.4$ & 0.14 \\
\hline
\end{tabular}



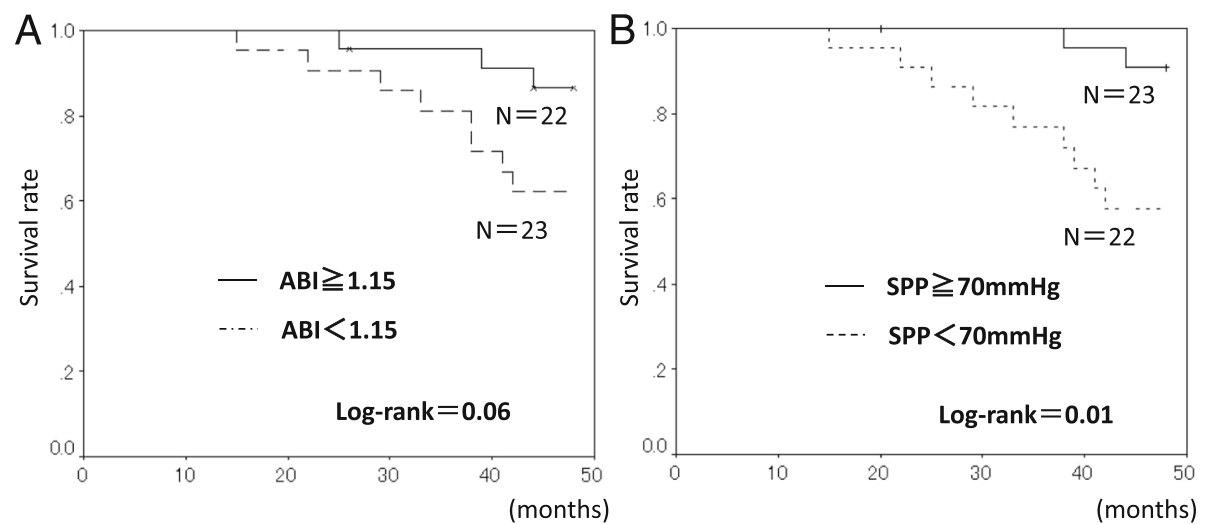

Fig. 1 Survival rate of comparison between higher and lower rate of ankle-brachial index (ABI) decline determined using Kaplan-Meier analysis (a) and SPP (b) are shown

\section{Discussion}

The patients with classic cardiovascular risk factors have usually polyvascular disease as PAD, coronary artery disease [20]. Especially in the patients undergoing HD, the presence of PAD is reported to increase morbidity and mortality significantly [21, 22]. Thus, early detection of PAD is an important issue for the HD patients to predict mortality [23]. ABI and SPP have been used for the screening of PAD in the HD patients clinically $[8,10]$. $\mathrm{ABI}$ has been widely used for detection of PAD by assessment of arterial stenosis of the lower extremities. $\mathrm{ABI}$ is used commonly and has a merit of the short testtime as compared with SPP. ABI as early prediction is particularly important for management of CLI in patients with suspected peripheral artery disease. [24] It is reported that $\mathrm{ABI}$ is an independent risk factor of cardiovascular events in PAD patients [25].

Table 3 Prognostic factors in the Cox proportional hazards model

\begin{tabular}{|c|c|c|c|c|}
\hline & \multicolumn{2}{|c|}{ Univariate analysis } & \multicolumn{2}{|c|}{ Multivariate analysis } \\
\hline & $p$ value & $\begin{array}{l}\text { Hazard ratio } \\
(95 \% \mathrm{Cl})\end{array}$ & $p$ value & $\begin{array}{l}\text { Hazard ratio } \\
(95 \% \mathrm{Cl})\end{array}$ \\
\hline Age (years) & 0.00 & $1.1(1.04-1.16)$ & 0.60 & $1.0(0.951-1.090)$ \\
\hline DM & 0.03 & $3.8(1.11-13.07)$ & 0.05 & $3.9(1.026-14.869)$ \\
\hline $\mathrm{SPP}<70 \mathrm{mmHg}$ & 0.02 & $6.0(1.29-27.83)$ & 0.02 & $7.2(1.329-39.04)$ \\
\hline $\mathrm{ABI}<1.15$ & 0.04 & $0.1(0.01-0.85)$ & 0.94 & $1.1(0.04-30.4)$ \\
\hline LVDd (mm) & 0.26 & $1.1(0.95-1.21)$ & & \\
\hline EF $(\%)$ & 0.00 & $0.9(0.85-0.96)$ & 0.00 & $0.86(0.79-0.94)$ \\
\hline $\mathrm{Ca}(\mathrm{mg} / \mathrm{dl})$ & 0.55 & $0.57(0.24-1.39)$ & & \\
\hline$P(\mathrm{mg} / \mathrm{dl})$ & 0.87 & $0.77(0.38-1.54)$ & & \\
\hline i-PTH (pg/dl) & 0.64 & $1.004(0.99-1.01)$ & & \\
\hline Alb (g/dl) & 0.04 & $0.3(0.09-0.94)$ & 0.14 & $0.39(0.11-1.38)$ \\
\hline $\mathrm{Hb}(\mathrm{g} / \mathrm{dl})$ & 0.85 & $1.1(0.56-2.01)$ & & \\
\hline
\end{tabular}

Since there have been no standardized values for SPP in the HD patients, some used SPP of $39 \mathrm{mmHg}$ for diagnosis of PAD as cutoff point $[10,26]$. However, they measured SPP of the dorsal artery of the foot and the endpoint of the study was the stenosis of the peripheral artery evaluated by CT scan, which is completely different from our study in both measurement site and endpoint. In our study for the HD patients without symptoms of PAD, the cutoff value of SPP was $61.8 \mathrm{mmHg}$ (sensitivity, 71\%; specificity, 65\%) according to ROC analysis. In this study, we used median values $70 \mathrm{mmHg}$ for the cutoff points of SPP. SPP lower group showed significantly more cardiac events to classify HD patients as two groups using $70 \mathrm{mmHg}$ as SPP cutoff point. We examined only ambulatory patients, excluding those cases with symptoms of PAD. Our results demonstrate that SPP would be useful for the evaluation of mortality risk in the HD patients since the mortality of the patients with lower SPP values is significantly higher.

This result is caused by the difference of ABI and SPP although both methods have been used for detection of PAD; the conditions of the larger artery are evaluated by ABI measurement while SPP can detect the state of subcutaneous microcirculation. The lower ABI values reflect the stenosis of the larger vessels but not of the peripheral smaller vessels. On the other hand, SPP is useful for detection of such patients with normal ABI values as may be suffered from stenosis of the peripheral smaller vessels though the larger arteries are not stenotic. ABI values are elevated as systemic calcification is frequently found in the HD patients, but SPP values are not influenced by calcification [27]. Therefore, it is possible that SPP can detect the presence of PAD more accurately than ABI and SPP values are supposed a risk factor predicting the prognosis of the HD patients without symptoms of PAD. 
In our study, nobody died of infection due to lower limb ischemia among 11 deaths. Two dead patients suffered from lower limb ulcers. Lower SPP value is supposed a risk factor of severe ischemic disease in the lower limb since the patients with higher SPP values do not suffer from ischemic ulcers. Previous study reported wound healing is impaired in the patients with SPP value of $30 \mathrm{mmHg}$ [18]. The preservation of microcirculation is important for the prevention of death from infection.

We analyzed risk factors to predict prognosis of the HD patient. As a result, SPP values as well as diabetic status and LVEF were independent risk factors by multivariate analysis. There have been many reports that the presence of diabetes increased mortality for the HD patients since diabetes was a risk factor for coronary artery disease, and diabetic patients suffered from infection due to severe ischemic PAD [8]. The patients with lower LVEF have higher risk for heart failure which is a frequent cause of death for the HD patients [28]. Furthermore, our results also indicated SPP values for important risk factors and hazard ratio of SPP values was higher than that of diabetes or LVEF. These results suggest that preservation of circulation in the peripheral smaller blood vessels in the lower extremities is important to improve the prognosis of the HD patients as well as circulation of the larger vessels. Furthermore, the screening of peripheral blood circulation by SPP is useful to those HD patients who showed decreased circulation in the peripheral vessels in the lower extremities. Since our study has a limitation of one single institute study with smaller number of HD patients, validation of multicenter study with larger number of patients will be required.

\section{Conclusions}

We studied the effectiveness of SPP to predict the prognosis of HD patients. The lower SPP group showed significantly worse survival curve than the higher SPP group while there were no differences between the higher and lower ABI groups. Cox proportional hazards model demonstrated SPP values, LVEF, and diabetes are independent and significant risk factors for mortality. These results suggest evaluation of PAD by SPP measurement is useful to predict prognosis of HD patients.

\section{Acknowledgements \\ This work was supported by making acquisition of data from Nagahara Hospital of Blood Purification Center, Osaka, Japan. This study was not funded by any organization.}

\section{Funding}

The study has no specific funding.

\section{Availability of data and materials}

No data has been submitted to any open access databases. All data supporting the study is presented in the manuscript or is available upon request.

\section{Authors' contributions}

NK carried out the conception and design, data collection, data analysis, and manuscript writing. YT, SM, SAl, and MN participated in its design and coordination of the manuscript. YH and NM conceived of the study, participated in its design and coordination, and helped to draft the manuscript. All authors read and approved the final manuscript.

\section{Competing interests}

The authors declare that they have no competing interests.

\section{Consent for publication}

Not applicable.

\section{Ethics approval and consent to participate}

Informed written and oral consent was obtained from all the participants prior to participation in the study.

\section{Author details}

'Department of Molecular Pathology, Osaka University Graduate School of Medicine and Health Science, 1-7, Yamadaoka, Suita, Osaka 565-0871, Japan. ${ }^{2}$ Blood Purification Center, Nagahara Hospital, 4-3-13 Nagatanishi, Higashiosaka, Osaka 577-0016, Japan.

Received: 26 November 2015 Accepted: 2 November 2016

Published online: 20 December 2016

\section{References}

1. Nakai S, Watanabe Y, Masakane I, et al. Overview of regular dialysis treatment in Japan (as of 31 December 2011). Ther Apher Dial. 2013;17: 567-611.

2. Herzog CA, Asinger RW, Berger AK, et al. Cardiovascular disease in chronic kidney disease. A clinical update from Kidney Disease: Improving Global Outcomes (KDIGO). Kidney Int. 2011;80:572-86.

3. Hirakata H, Nitta K, Inaba M, et al. Japanese Society for Dialysis Therapy guidelines for management of cardiovascular diseases in patients on chronic hemodialysis. Ther Apher Dial. 2012;16:387-435.

4. Moe S, Drueke T, Cunningham J, et al. Definition, evaluation, and classification of renal osteodystorophy: a position statement from Kidney Disease: Improving global outcomes (KDIGO). Kidney Int. 2006;69:1945-53.

5. O'Hare AM, Hsu CY, Bacchetti P, et al. Peripheral vascular disease risk factors among patients undergoing hemodialysis. J Am Soc Nephrol. 2002;13: 497-503.

6. Garimella PS, Hart PD, O'Hare AM, et al. Peripheral artery disease and CKD: a focus on peripheral artery disease as a critical component of CKD care. Am J Kidney Dis. 2012;60:641-54.

7. Menke A, Muntner P, Wildman RP, et al. Relation of borderline peripheral arterial disease to cardiovascular disease risk. Am J Cardiol. 2006;98:1226-30

8. Rajagopalan S, Dellegrotaglie S, Furniss AL, et al. Peripheral arterial disease in patients with end-stage renal disease: observations from the Dialysis Outcomes and Practice Patterns Study (DOPPS). Circulation. 2006;114: 1914-22.

9. Yoshitaka K, Toru A, Hideki I, et al. Long-term outcome of percutaneous transluminal angioplasty in chronic haemodialysis patients with peripheral arterial disease. Nephrol Dial Transplant. 2008;23:3996-4001.

10. Okamoto $\mathrm{K}$, Oka M, Maesato $\mathrm{K}$, et al. Peripheral arterial occlusive disease is more prevalent in patients with hemodialysis: comparison with the findings of multidetector-row computed tomography. Am J Kidney Dis. 2006;48:269-76.

11. Jaar BG, Astor BC, Bems JS, et al. Predictors of amputation and survival following lower extremity revascularization in hemodialysis patients. Kidney Int. 2004;65:613-20.

12. Jaccard $Y$, Walther $S$, Anderson $S$, et al. Influence of secondary infection on amputation in chronic critical limb ischemia. Eur J Vasc Endovasc Surg. 2007:33:605-9.

13. Diehm C, Allenberg JR, Pittrow D, German Epidemiological Trial on Ankle Brachial Index Study Group, et al. Mortality and vascular morbidity in older adults with asymptomatic versus symptomatic peripheral artery disease. Circulation. 2009;120:2053-61.

14. Bird CE, Criqui MH, Fronek A, et al. Quantitative and qualitative progression of peripheral arterial disease by non-invasive testing. Vasc Med. 1999;4:15-21. 
15. Ogata H, Kumata-Maeta C, Shishido K, et al. Detection of peripheral artery disease by duplex ultrasonography among hemodialysis patients. Clin J Am Soc Nephron. 2010;5:2199-206.

16. Ohtake T, Kobayashi S, Moriya H, et al. High prevalence of occult coronary artery stenosis in patients with chronic kidney disease at the initiation of renal replacement therapy: an angiographic examination. J Am Soc Nephrol. 2005;16:1141-8.

17. Castronuovo JJ, Adera H, Smiell JM, et al. Skin perfusion pressure measurement is valuable in the diagnosis of critical limb ischemia. J Vascular Surgery. 1997;26:629-37.

18. Adera HM, James K, Castronuovo JJ, et al. Prediction of amputation wound healing with skin perfusion pressure. J Vasc Surg. 1995;21:823-8.

19. Tsai FW, Tulsyan N, Jones DN, et al. Skin perfusion pressure of the foot is a good substitute for toe pressure in the assessment of limb ischemia. J Vasc Surg. 2000;32:32-6.

20. Bhatt DL, Steq PG, Ohman EM, et al. International prevalence, recognition, and treatment of cardiovascular risk factors in outpatients with atherothrombosis. JAMA. 2006;11:180-9.

21. Adragao T, Pires A, Branco P, et al. Ankle-brachial index, vascular calcifications and mortality in dialysis patients. Nephrol Dial Transplant. 2012;27:318-25.

22. Otsubo $\mathrm{S}$, Kitamura $\mathrm{M}$, Wakaume T, et al. Association of peripheral artery disease and long-term mortality in hemodialysis patients. Int Urol Nephrol. 2012;44:569-73.

23. Hoffmann F, Haastert B, Koch M, et al. The effect on incidence and mortality in end-stage renal disease in Germany. Nephrol Dial Transplant. 2011;26: 1634-40.

24. Yoshikawa H, lijima R, Hashimoto G, et al. Prediction of development of critical limb ischemia in hemodialysis patients. Ther Apher Dial. 2015;19: 378-84.

25. Thatipelli MR, Pellikka PA, McBane RD, et al. Prognostic value of anklebrachial index and dobutamine stress echocardiography for cardiovascularmorbidity and all-cause mortality in patients with peripheral arterial disease. J Vasc Surg. 2007;46:62-70.

26. Okamoto K, Oka M, Maesati K, et al. Non-invasive diagnostic modality for peripheral arterial occlusive disease in hemodialysis patients. Angiology. 2006:46:829-35

27. Kitaura K, Kida M, Hirama K. Assessment of peripheral arterial disease of lower limbs with ultrasonography and ankle brachial index at the initiation of hemodialysis. Ren Fail. 2009;31:785-90.

28. Yamada $\mathrm{S}$, Ishii $\mathrm{H}$, Takahashi $\mathrm{H}$, et al. Prognostic value of reduced left ventricular ejection fraction at start hemodialysis therapy on cardiovascula and all-cause mortality in end-stage renal disease patients. Clin J Am Soc Nephrol. 2010;5:1793-8.

\section{Submit your next manuscript to BioMed Central and we will help you at every step:}

- We accept pre-submission inquiries

- Our selector tool helps you to find the most relevant journal

- We provide round the clock customer support

- Convenient online submission

- Thorough peer review

- Inclusion in PubMed and all major indexing services

- Maximum visibility for your research

Submit your manuscript at www.biomedcentral.com/submit

C) Biomed Central 